\title{
Arthur Richs Bedeutung für die Entwicklung der Wirtschaftsethik ${ }^{*}$
}

\author{
GEORGES ENDERLE ${ }^{* *}$
}

\section{Arthur Rich's Importance for the Development of Business and Economic Ethics}

Arthur Rich's fundamental and unique masterpiece 'Business and Economic Ethics. The Ethics of Economic Systems' (published in German in 1984/1990; in English in 2006) is discussed against the backdrop of the international development of business and economic etbics since the 1980s. A great number of important insights are still relevant for the foundation of business and economic ethics and the development of a bumanly reformed and ecologically regulated market economy: the contribution of Christian theology to the etbical dialogue in a pluralistic society; Rich's etbical reasoning that defies ethical relativism and skepticism; Rich's "bermeneutic circle" of economic rationality and ethical reasoning; the distinction of economic systems and economic orders and the variety of market economies; the indispensable ecological dimension of any market economy; basic conditions for a participative cooperation in a global economy, etc.

Keywords: Foundation of Business and Economic Ethics, a Christian Perspective in a Pluralist Society; "Critical Hermeneutics" of Economic Rationality and Ethical Reasoning, the Ethics of Economic Systems, Toward a Humanly Reformed and Ecologically Regulated Market Economy.

\section{Einleitung}

Arthur Richs Leben und Werk zu feiern ist eine noble und interessante Aufgabe, wozu uns die Veranstalter dieser Gedenktagung eingeladen haben. ${ }^{1}$ Ich nehme diese Einladung dankbar an und fühle mich geehrt, dass ich einen Beitrag zu dieser Würdigung vorlegen darf. Sie ist für mich auch ein wichtiger Anstoß, über die Entwicklung der Wirtschaftsethik im Licht von Richs grundlegender Arbeit nachzudenken.

Kurz nach Arthur Richs Hinschied im Sommer 1992 verließ ich die Schweiz, um eine Professur in internationaler Wirtschafts- und Unternehmensethik an der Universität

Beitrag eingereicht am 05.02.2010; nach doppelt verdecktem Gutachterverfahren überarbeitete Fassung angenommen am 10.09.2010.

* $\quad$ Prof. Dr. Georges Enderle, Department of Marketing, Mendoza College of Business, University of Notre Dame, Notre Dame, Indiana 46566-0399, USA, Tel.: +1 574 631-5595, Fax: +1 574 631-5255, E-Mail: genderle@nd.edu, Forschungsschwerpunkte: Ethik der Globalisierung, Schaffung von Reichtum (wealth creation), Unternehmensverantwortung und Menschenrechte, wirtschafts- und unternehmensethische Entwicklungen in China.

1 Die Tagung zum 100. Geburtstag von Arthur Rich Theologische Wirtschaftsetbik. Stand, Aufgaben und Perspektiven fand am 22. Januar 2010 an der Theologischen Fakultät der Universität Zürich statt, gefolgt von einer Buchvernissage von Walter Wolfs (2009) neuem Buch Für eine sozial verantwortbare Marktwirtschaft. Der Wirtschaftsetbiker Arthur Rich. 
von Notre Dame in den USA zu übernehmen. Ich hatte das außerordentliche Privileg, in diesem sich rasch entwickelnden Gebiet vollzeitig zu forschen und zu lehren und unter dem Druck der Globalisierung in zahlreichen Ländern tätig zu sein. In diesen fast 20 Jahren habe ich immer deutlicher realisiert, wie sehr mein Denken und Tun von der vortrefflichen Erziehung und Ausbildung beeinflusst worden war, die ich in der Schweiz und Europa erfahren durfte. Für meine wirtschaftsethische Arbeit war Arthur Rich von entscheidender Bedeutung. Durch sein persönliches Beispiel und sein weitblickendes und gründliches wissenschafliches Werk hat er mir und meiner Generation von Kollegen und Kolleginnen vorgelebt, dass Wirtschaftsethik in Theorie und Praxis möglich und kein Widerspruch in sich selbst (oder ein sogenanntes ,Oxymoron') ist. Aber nicht nur das; Rich zeigte uns auch die Notwendigkeit und Dringlichkeit der Wirtschaftsethik und auf welche Weise wir dieses komplexe Gebiet aus christlicher Perspektive angehen und behandeln können. In der Tat wäre meine Arbeit und die Arbeit vieler in Wirtschaftsethik ohne Richs Pionierleistung nicht möglich gewesen. Wir stehen sozusagen auf seinen Schultern und wissen uns zu großem Dank verpflichtet.

Im Folgenden möchte ich Richs Werk im Kontext der internationalen Entwicklung der Wirtschaftsethik seit den achtziger Jahren und im Ausblick auf die nächsten Jahre diskutieren und dabei auch auf theologische Aspekte zu sprechen kommen.

\section{Konturen der internationalen Entwicklung der Wirtschaftsethik}

Es ist nicht leicht, die Konturen der internationalen Entwicklung der Wirtschaftsethik seit den achtziger Jahren nachzuzeichnen. Nicht nur ist eine unübersehbare Fülle von Initiativen, Veröffentlichungen, Organisationen und Institutionen entstanden. Diese Entwicklungen haben auch in verschiedenen Ländern, Sprachen und Kulturen stattgefunden, weshalb es verfehlt wäre, nur die englische Literatur in Betracht zu ziehen (was jedoch leider allzu oft geschieht). Überdies sind sehr unterschiedliche Verständnisse und Definitionen der ,Wirtschaftsethik' in Gebrauch gekommen, die internationale Vergleiche noch erschweren. Aus diesen Gründen wird meine Darstellung notwendigerweise begrenzt und von meinen Erfahrungen beeinflusst sein. Ich werde mich hauptsächlich auf Übersichtsartikel, Lexika, Surveys und andere Standardwerke beziehen (siehe Abbildung 1).

\begin{tabular}{|c|c|c|}
\hline \multicolumn{3}{|c|}{ Zur internationalen Entwicklung der Wirtschaftsethik } \\
\hline Zeitschriften & $\begin{array}{l}\text { - Journal of Business Ethics } \\
\text { (seit 1982) } \\
\text { - Economics and Philosophy } \\
\text { (seit 1985) } \\
\text { - Etica degli affari [e delle } \\
\text { professioni] (1988-1991) } \\
\text { - Revue éthique des affaires } \\
\text { - } 1995-1998) \\
\text { - Business Ethics Quarterly } \\
\text { (seit 1991) }\end{array}$ & $\begin{array}{l}\text { - Journal of Human Values } \\
\text { (seit 1995) } \\
\text { - Teaching Business Ethics } \\
\text { (1997-2003) } \\
\text { - Business and Society Review } \\
\text { (seit 1999) } \\
\text { - Zeitschrift für Wirtschafts- } \\
\text { und Unternehmensethik } \\
\text { (seit 2000) } \\
\text { - Journal of Business Ethics } \\
\text { Education (seit 2004) }\end{array}$ \\
\hline
\end{tabular}




\begin{tabular}{|c|c|c|}
\hline & $\begin{array}{l}\text { - Business Ethics - A } \\
\text { European Review (seit 1992) } \\
\text { - Business and Society (seit } \\
\text { 1994) }\end{array}$ & $\begin{array}{l}\text { African Journal of Business } \\
\text { Ethics (seit 2005) } \\
\text { - Journal of International } \\
\text { Business Ethics (seit 2008) }\end{array}$ \\
\hline Gesellschaften & $\begin{array}{l}\text { - (American) Society of } \\
\text { Business Ethics, SBE (seit } \\
\text { 1980) } \\
\text { - European Business Ethics } \\
\text { Network, EBEN (seit 1987) } \\
\text { - International Society of } \\
\text { Business, Economics, and } \\
\text { Ethics, ISBEE (seit 1989) } \\
\text { - Verein für Socialpolitik: } \\
\text { Ausschuss "Wirtschafts- } \\
\text { wissenschaften und Ethik" } \\
\text { (seit 1989) } \\
\end{array}$ & $\begin{array}{l}\text { - International Association of } \\
\text { Business and Society, IABS } \\
\text { (seit 1990) } \\
\text { - The Japanese Society for } \\
\text { Business Studies (seit 1996) } \\
\text { - The Business Ethics } \\
\text { Network of Africa, BEN- } \\
\text { Africa (seit 1999) } \\
\text { - Asociación Latinoamericana } \\
\text { de Ética en los Negocios y } \\
\text { Economía, ALENE (seit } \\
\text { 2000) }\end{array}$ \\
\hline Lexika, etc. & $\begin{array}{l}\text { Lexikon der } \\
\text { Wirtschaftsethik (1993), } \\
\text { Hrsg. G. Enderle, K. } \\
\text { Homann, W. Kerber, M. } \\
\text { Honecker, H. Steinmann } \\
\text { - Encyclopedia of Business } \\
\text { Ethics (1997; 2. Aufl. 2005), } \\
\text { Hrsg. P. H. Werhane, R. E. } \\
\text { Freeman } \\
\text { - Handbuch der } \\
\text { Wirtschaftsethik (1999), } 4 \\
\text { Bände, Gesamtleitung: W. } \\
\text { Korff, S. Feldhaus } \\
\text { - A Companion to Business } \\
\text { Ethics (1999), Hrsg. R. E. } \\
\text { Frederick }\end{array}$ & $\begin{array}{l}\text { Encyclopedia of Business } \\
\text { Ethics and Society (2008), } 5 \\
\text { Bände, Hrsg. R. W. Kolb } \\
\text { - The Oxford Handbook of } \\
\text { Corporate Social } \\
\text { Responsibility (2008), Hrsg. } \\
\text { A. Crane, A. McWilliams, D. } \\
\text { Matten, J. Moon, D. S. } \\
\text { Siegel } \\
\text { - Handbook of Research on } \\
\text { Global Corporate } \\
\text { Citizenship (2008), Hrsg. A. } \\
\text { G. Scherer, G. Palazzo } \\
\text { - The Oxford Handbook of } \\
\text { Business Ethics (2010), } \\
\text { Hrsg. G. G. Brenkert, T. L. } \\
\text { Beauchamp }\end{array}$ \\
\hline Surveys & $\begin{array}{l}\text { - Weltweiter Survey in: } \\
\text { Journal of Business Ethics, } \\
\text { Vol. 16/No. } 14 \\
\text { - Nordamerika und } \\
\text { Kontinentaleuropa in: } \\
\text { Business Ethics - A } \\
\text { European Review, Vol. } \\
\text { 4/No. } 2 \\
\text { - Business Ethics. In: } \\
\text { Blackwell Companion to } \\
\text { Philosophy (2003) }\end{array}$ & $\begin{array}{l}\text { - Business Ethics: Europe } \\
\text { versus America (D. Melé } \\
\text { 2008) } \\
\text { - Länderberichte in: } \\
\text { Encyclopedia of Business } \\
\text { Ethics } 2005 \\
\text { - Globaler Survey 2009-2010, } \\
\text { durchgeführt von } \\
\text { Globethic.net }\end{array}$ \\
\hline
\end{tabular}

Abbildung 1: Zur internationalen Entwicklung der Wirtschaftsethike 
Unter dem Einfluss der internationalen Diskussion hat sich eine Konzeption der Wirtschaftsethik eingebürgert, die den Untersuchungsbereich mit Hilfe von drei Ebenen strukturiert und die Zuordnung der Verantwortlichkeiten erleichtert:

(1) die Entscheidungsträger und Akteure auf der individuellen (oder Mikro-) Ebene: Manager, Angestellte, Aufsichtsräte, Konsumentinnen und Konsumenten, Investoren und viele mehr;

(2) Organisationen als Entscheidungsträger und Akteure auf der Meso-Ebene: Unternehmen, Investitionsgesellschaften, Berufsorganisationen (von Revisoren, Finanzexperten und anderen), Rating-Agenturen, Konsumentenorganisationen, Gewerkschaften, etc.;

(3) die systemische (oder Makro-) Ebene, wo die Grundregeln der Wirtschaft für einzelne Länder und transnationale Einheiten festgelegt werden (vgl. Enderle 2003).

Gemäß dieser Konzeption umfasst also der deutsche Begriff der Wirtschaftsethik alle drei Ebenen, das heißt, den gesamten Lebensbereich der Wirtschaft, wobei die Unternehmensethik nur den Meso-Bereich abdeckt (siehe Abbildung 2). Ähnlich verhält es sich mit dem chinesischen Begriff ,jingji lunli xue‘, dem französischen ,éthique économique ${ }^{\varsigma}$ und dem spanischen ,ética económica‘. (Unternehmensethik, auch verkürzt Unternehmensverantwortung genannt, heißt entsprechend, shangye lunli xue', ethique de l'entreprise' und ,ética de la empresa'). Im Englischen ist der Sprachgebrauch unklarer, weil schon der Begriff ,business ${ }^{6}$ mindestens vier Bedeutungen enthält (siehe ,the business of business is business' und ,business cycles ${ }^{9}$. Wenn wir demnach von ,business ethics` reden, ist es ratsam, nachzufragen, was damit genauer gemeint ist: die Ethik von Individuen, die ,business' betreiben; oder die Ethik von ,business organizations'; oder die Ethik der (Gesamt-) Wirtschaft. Um mögliche Missverständnisse zu vermeiden, haben wir deshalb Richs ,Wirtschaftsethik' ${ }^{`}$ mit , business and economic ethics ${ }^{`}$ übersetzt.

\begin{tabular}{|c|l|}
\hline \multicolumn{2}{|c|}{ Definitionen von Wirtschaftsethik / Business Ethics } \\
\hline & $\begin{array}{l}\text { 'Business ethics' is understood in two distinctive ways by those who } \\
\text { have a major interest in its development: } \\
\text { The philosophical ethics orientation consists of the analysis of moral } \\
\text { problems and case studies using categories such as justice, utility, } \\
\text { Handbook } \\
\text { of Business } \\
\text { Ethics (2010) } \\
\text { rights, obligations, and personal virtues. } \\
\text { The business orientation tends to view its endeavor in terms of vari- } \\
\text { ous relationships that obtain largely within business: employer and } \\
\text { employee, manager and workers, supervisors and supervisees. }\end{array}$ \\
\hline dtv-Lexikon & $\begin{array}{l}\text { Wirtschaftsethik ist der Zweig der Sozialethik, der sich um systemati- } \\
\text { sche Verarbeitung der wirtschaftswissenschaftlichen Forschung und } \\
\text { (2006) }\end{array}$ \\
& $\begin{array}{l}\text { der ökonomischen Wirklichkeit im Horizont ethischen Denkens } \\
\text { bemä. }\end{array}$ \\
\hline
\end{tabular}




\begin{tabular}{|l|l|}
\hline & $\begin{array}{l}\text { Besonders wichtige Probleme sind die Reflexion über moralische } \\
\text { Aspekte des wirtschaftlichen Handelns, insbesondere über das Ei- } \\
\text { gentum, die Mitbestimmung, das Verhältnis von Lohn und Arbeit, } \\
\text { Vollbeschäftigung, Rüstung, die Technik, Wirtschaftswachstum, } \\
\text { ökologische bzw. Energie- und Rohstoffkrise. }\end{array}$ \\
\hline
\end{tabular}

Abbildung 2: Definitionen von Wirtschaftsethik / Business Ethics

\section{Zur Relevanz des ersten Bandes Grundlagen der Wirtschaftsethik in theologischer Perspektive}

Im ersten Band werden ,die Grundlagen der Wirtschaftsethik in theologischer Perspektive“ erarbeitet, die eine solide Basis für die Behandlung der vielfältigen Probleme auf der Mikro-, Meso- und Makro-Ebene bilden (Rich 1984: 60f.). Der Band ist ein Meisterwerk an Klarheit, Tiefe und Weite des ethischen Denkens und macht in überzeugender Weise deutlich, dass ,gewissenhafte Ethik” nie abgeschlossen ist und immer Aufgabe bleibt (Rich 1984: 19). Er sollte Pflichtlektüre für alle Studierenden und auch Lehrenden der Wirtschafts- und Sozialethik sein und hat auch tatsächlich eine breite Rezeption im deutschsprachigen Raum (mit vier Auflagen seit 1984) erlebt.

Aus den Grundlagen möchte ich vier Aspekte hervorheben, die mir im Kontext der internationalen Entwicklung der Wirtschaftsethik besonders wichtig erscheinen.

\subsection{Richs Wirtschaftsethik versteht sich als ein christlicher Beitrag in plura- listischer Gesellschaft.}

Wie nur sehr wenige Autoren der Wirtschaftsethik behandelt Rich das Spannungsverhältnis von christlichem Glauben und säkularisierter Gesellschaft in ausdrücklicher und sehr differenzierter Weise. Er verschweigt nicht seine Identität, Herkunft und Motivationskraft und klammert sie nicht aus; vielmehr bekennt er sich dazu in ehrlicher und bescheidener Bestimmtheit. Gleichzeitig nimmt er auch die Herausforderung sehr ernst, dass keine Religion und kein philosophischer Glaube den Bürgern und Bürgerinnen in autoritärer Manier aufgezwungen werden darf. Was aus christlicher Perspektive in den Dialog mit Gläubigen und Nichtgläubigen eingebracht wird, muss für alle nachvollziehbar sein.

Für mich ist eine solche Haltung von zukunftsweisender Bedeutung. Leider hat sich die wirtschaftsethische Diskussion bisher viel zu wenig mit der Rolle und Verantwortung der Religionen in der Wirtschaft auseinandergesetzt. Wir sind immer noch zu sehr von der falschen Alternative blockiert, dass das Religiöse entweder verschwiegen oder aufgezwungen werden müsse. Eine freie Diskussion scheint (noch) nicht möglich zu sein. So finden wir in der wirtschaftsethischen Literatur der achtziger und neunziger Jahre nur spärliche Veröffentlichungen zu dieser Thematik, trotz einigen kirchlichen Verlautbarungen, internationalen Erklärungen (wie Toward a Global Ethic 1992, International Business Ethics 1994 und Stiftung Weltethos) und 
Schwerpunktthemen an internationalen Konferenzen (wie an den Weltkongressen der International Society of Business, Economics and Ethics von 1996 und 2000). Die führenden wirtschaftsethischen Zeitschriften (Journal of Business Ethics, Business Ethics Quarterly, Business Ethics - A European Review, Business and Society und andere) und die meisten Lexika und Lehrbücher haben religiöse Fragen der Wirtschaftsethik bisher nur sehr sporadisch behandelt. ${ }^{2}$

Im Gegensatz dazu sind die Stimmen der religiösen und ideologischen Fundamentalisten, besonders nach dem 11. September 2001, immer lauter geworden. Viele Wirtschaftsethiker scheinen Mühe zu haben, diesen Herausforderungen angemessen zu begegnen, weil sie unvorbereitet sind oder die religiöse Thematik für nebensächlich halten. Zur Illustration dieses schwierigen Sachverhalts kann ich auf eine Konferenz an meiner Universität im Jahr 2007 verweisen, an der wir einen wirtschaftsethischen Dialog zwischen Juden, Christen und Moslems versuchten. Trotz des guten Willens auf allen Seiten erbrachte das Gespräch jedoch nicht viel, mit Ausnahme der Einsicht, dass der Weg vor uns noch sehr lang ist. Mit Arthur Richs Ansatz, Gründlichkeit und Geduld ist es aber möglich, diesen Weg mit Zuversicht zu gehen.

\subsection{Richs Vernunftethik widersteht weitverbreitetem ethischen Relativismus und Skeptizismus.}

Trotz der beachtlichen Fortschritte, die die Wirtschaftsethik seit den achtziger Jahren in vielen Ländern gemacht hat, ist es erstaunlich, dass an vielen Universitäten und besonders in den Wirtschafts- und Sozialwissenschaften noch ein ethischer Relativismus oder gar Skeptizismus herrscht. In vielen akademischen Kreisen ist die Ethik immer noch eine reine Gefühlssache, eine private Angelegenheit oder ein bloßes gesellschaftlich-kulturelles Konstrukt, worüber man keine vernunftmäßig abgesicherten Werturteile fällen kann und darf. Für diese Leute hat die Ethik keine wissenschaftliche Legitimität. Dies zeigt sich zum Beispiel am oft heftigen Widerstand an Universitäten, Ethik in Form von Forschungsinstituten, Lehrstellen und Lehrplänen zu institutionalisieren. Es wird auch sichtbar bei der Besetzung von Lehrstühlen für Wirtschaftsethik, wenn gründliche Kenntnis in Ethik weder von der Berufungskommission noch von den Kandidaten verlangt wird - eine Situation, die leider öfters eingetreten ist. Ich bin sicher, dass für Arthur Rich diese selbstgewollte Ignoranz unverständlich ist.

\subsection{Richs Grundlegung der Ethik entspringt christlicher Tugendethik und entwickelt universelle Kriterien der Humanität.}

Wie schon erwähnt, behandelt Rich das Spannungsverhältnis von christlichem Glauben und säkularisierter Gesellschaft in ausdrücklicher und sehr differenzierter Weise. Es ist nun interessant, genauer zu verstehen, wie er dies bewerkstelligt. Im Unterschied zu den meisten Wirtschaftsethikern der achtziger bis in die neunziger

2 Es ist erfreulich, dass das Deutsche Netzwerk Wirtschaftsethik (DNWE) für die Jahrestagung 2010 das Thema Religion. Störfaktor und Ressource in der Wirtschaft gewählt hat. 
Jahre legte Rich keine Prinzipienethik zugrunde, wie es etwa die Kantianer oder Utilitaristen tun. Vielmehr wählte er als Quelle und Ausgangspunkt die drei christlichen theologischen Tugenden des Glaubens, der Hoffnung und der Liebe. Er machte damit nicht den Fehler, den Alasdair MacIntyre den zeitgenössischen Ethikern in seinem Buch After Virtue von 1981 (MacIntyre 1981) vorwarf, nämlich dass die modernen Ethiken seit der Aufklärung die jahrhundertealte Tradition der Tugendethik über Bord geworfen hätten. Seit den späten neunziger Jahren hat dann bekanntlich die Tugendethik - neben der Prinzipienethik - einen festen Platz in der Wirtschaftsethik bekommen. Und selbst eine eingeschworene Ökonomin der Chicago Schule, Deirdre McCloskey, hat es in ihrem groß angelegten Werk unternommen, die Tugendethik zu rehabilitieren. Der erste Band über „Die bürgerlichen Tugenden“ (McCloskey 2006) erschien 2006, und drei weitere Bände sollen folgen. Sie kritisiert die herrschende Auffassung der Wirtschaftswissenschaften, dass mit dem Ziel der Maximierung des Selbstinteresses nur die Tugend der Klugheit maßgeblich sei. Dabei seien die anderen drei traditionellen Kardinaltugenden der Gerechtigkeit, Tapferkeit und Mäßigung sowie die drei theologischen Tugenden des Glaubens, der Hoffnung und der Liebe auf der Strecke geblieben. In Tat und Wahrheit müssten aber alle sieben Tugenden in deren gegenseitigen Verwiesenheit (oder Relationalität, wie Rich sagen würde) und als Ganzes verstanden werden.

Als ich McCloskey letztes Jahr auf einer Konferenz fragte, wie nun diese tugendethische Grundlage - über die Mikro-Ebene hinaus - auf Organisationen (auf der Meso-Ebene) und Systeme (auf der Makro-Ebene) angewandt werden soll, konnte sie mich nur auf die noch zu schreibenden weiteren Bände vertrösten. Arthur Rich aber hat einen wesentlichen Schritt schon vor über 25 Jahren gemacht. Seine sieben Kriterien der Geschöpflichkeit, kritischen Distanz, relativen Rezeption, Relationalität, Mitmenschlichkeit, Mitgeschöpflichkeit und Partizipation entspringen zwar aus den drei theologischen Tugenden, sind aber in ihrem Gehalt zugänglich und nachvollziehbar sowohl für Christen wie für Nichtchristen und können auch auf der Meso- und Makro-Ebene Anwendung finden. Mit anderen Worten, Rich hat der Wirtschaftsethik einen Weg bereitet, der sich der pluralistischen Herausforderung stellt (was von MacIntyre's Ansatz nicht gesagt werden kann); der für den gesamten Lebensbereich der Wirtschaft anwendungsfähig ist und der schon vor zwei Jahrzehnten auf die Frage des Wirtschaftssystems angewandt wurde (was McCloskey noch zu leisten hat).

\subsection{Richs Ansatz integriert das Menschengerechte und Sachgemäße in der Maximenbildung.}

Wirtschaftsethik wird oft als eine Art ,angewandter Ethik' bezeichnet, vergleichbar mit Bioethik, Medienethik, Entwicklungsethik und anderen mehr. Dabei kann das, was mit ,anwenden' gemeint ist, sehr verschiedene Bedeutungen annehmen. Nach einem simplen Verständnis werden absolute ethische Prinzipien und Werte dem Anwendungsbereich sozusagen aufgezwungen, ohne dass die Beschaffenheit dieses Bereichs in irgendeiner Weise relevant wäre. Ebenso simpel ist die andere Auffassung, wonach der ethische Gehalt von Prinzipien und Werten in der Anwendung sozusagen aufgeht und verschwindet. Was ein differenziertes Verständnis von Anwendung 
beinhalten könnte, ist jedoch in der Literatur auffallenderweise wenig diskutiert worden. Ed Freemans Kritik an der ,separation thesis', wonach Ethik und Sachbereich zwei völlig getrennte Bereiche seien, ist zwar berechtigt; sie trägt aber nicht viel dazu bei, die ethische und sachliche Dimension eines wirtschaftsethischen Problems zu unterscheiden und deren gegenseitige Verwiesenheit besser zu verstehen. Ein wichtiger konstruktiver Beitrag ist der neulich erschienene Band Public Reason and Applied Ethics: The Ways of Practical Reason in a Pluralist Society (Cortina et al. 2008). In ihm werden verschiedene Varianten des Anwendungsverständnisses diskutiert und der Ansatz der „critical hermeneutics“ vorgeschlagen (J. Conill in Cortina et al. 2008: 8598). Danach soll die normativ-ethische und die deskriptiv-analytische Dimension eines Problems in einem zirkulären Prozess erhellt und in ihrer gegenseitigen Verwiesenheit erfasst werden. In ähnlicher Weise hat Hilary Putnam die Fact-Value-Relation am Beispiel von Amartya Sens Development as Freedom (Sen 1999) diskutiert (Putnam 2002).

Richs Verständnis der Maximenbildung kann in diesem Licht gesehen werden. Er fasst es mit dem prägnanten Grundsatz zusammen:

„Es kann nicht wirklich menschengerecht sein, was nicht sachgemäß ist; und es kann nicht wirklich sachgemäß sein, was dem Menschengerechten widerstreitet“ (Rich 1984: 81).

Ethische Argumentation und sachgemäßes Verständnis der Wirtschaft sind aufeinander angewiesen, damit hilfreiche und tragfähige Maximen oder Grundsätze für wirtschaftliches Handeln und wirtschaftliche Institutionen erarbeitet werden können. Man kann sagen, Wirtschaftsethik muss ,auf zwei Beinen gehen', um ihre Aufgabe verantwortlich erfüllen zu können (vgl. Enderle 2003). Wird nur eines der beiden Beine benutzt, humpelt man dahin und kommt nicht richtig vorwärts.

Soviel zu Richs Grundlagen der Wirtschaftsethik in theologischer Perspektive. Sie sind der internationalen Entwicklung der Wirtschaftsethik in mancherlei Hinsicht vorausgeeilt.

\section{Zur Entwicklung der Unternehmensethik}

Wie wir wissen, hat Rich nach Veröffentlichung der Grundlagen in 1984 in harter Arbeit und vorgerücktem Alter den zweiten Band Marktwirtschaft, Planwirtschaft, Weltwirtschaft (Rich 1990) über die Ethik des Wirtschaftssystems geschrieben. Darin konnte er sein langjähriges Forschen während des Kalten Krieges in einer meisterhaften Synthese zusammenfassen. Kurz nach dem Fall der Berliner Mauer publizierte er den Band, in dem er die Grundlagen auf zentrale Fragen der MakroEbene anwandte. Dabei war er sich klar bewusst, dass er einen dritten Band über die Unternehmensethik, das heißt, die Anwendung der Grundlagen auf Fragen der MesoEbene, nicht mehr verfassen konnte. In seiner Bescheidenheit fragte er sich dann zuweilen, ob der zweite Band nicht zu spät kam (weil die Marktwirtschaft über die Planwirtschaft offenbar gesiegt hatte) und ob der dritte Band im Zeitalter der Globalisierung und transnationalen Unternehmen nicht immer wichtiger würde.

Die internationale Entwicklung der Wirtschaftsethik hat nach meiner Ansicht Rich insofern recht gegeben, als die Unternehmensethik wegen der rasanten Machtentfaltung der Konzerne und der Globalisierung außerordentlich wichtig geworden ist. Ich möchte aber zugleich festhalten, dass die Makro-Fragen des Wirtschaftssystems und der Wirtschaftsordnung nicht nur relevant geblieben sind, sondern in den letzten 
Jahren an Bedeutung sogar zugenommen haben. Im Folgenden werde ich deshalb zuerst einige Meso-Fragen diskutieren und anschließend auf ein paar Makro-Fragen eingehen.

Unternehmensethik wird im angelsächsischen Raum oft als ,business ethics ‘ bezeichnet, obschon dieser Begriff nicht eindeutig klar ist und auch eine breitere Bedeutung im Sinn von Wirtschaftsethik haben kann. Andere Bezeichnungen sind ,corporate ethics', ,CSR' oder ,corporate social responsibility', einfach ,corporate responsibility', ,corporate accountability and transparency', ,corporate citizenship', ,corporate sustainability', social entrepreneurship' und andere mehr. Auch der Begriff des Unternehmens kann verschiedenste Bedeutungen annehmen und damit das Verständnis der Unternehmensethik maßgeblich beinflussen. Wenn die Firma als simple Produktionsfunktion, ,nexus of contracts', ,powerful animal' oder ,amoral mechanism' konzipiert wird, kann es keine eigentliche Unternehmensethik geben. Wird das Unternehmen jedoch als eine menschliche Organisation mit einer speziellen Identität, Mission und Kultur und einem gewissen Handlungsspielraum verstanden, ist es sinnvoll, von Unternehmensethik zu sprechen.

Nun ist die Frage der Unternehmensethik nicht einfach eine abstrakte Frage, die so oder anders beantwortet werden kann. Vielmehr ist der Ruf nach Unternehmensethik aus den gesellschaftlichen Erwartungen entstanden, dass mächtige Organisationen über ihren rechtlich-wirtschaftlichen Bereich hinaus Einfluss auf Gesellschaft und Umwelt ausüben, wofür sie ethische Verantwortung übernehmen müssen. Darüber hinaus haben sich Unternehmen selber zu ethischem Verhalten verpflichtet, sei es aus innerer Überzeugung und/oder aus klugen Geschäftsüberlegungen heraus. Auch rechtliche Entwicklungen in den USA und vielen anderen Ländern haben zu der Auffassung geführt, dass Unternehmen neben der rechtlichen auch eine ethische Verantwortung für ihr Tun und Lassen tragen. Wie Professor Lynn Paine von der Harvard Business School in ihrer historischen Analyse des Unternehmensverständnisses in den USA schreibt, ist heute die Doktrin des Amoralismus (oder der Morallosigkeit, ,amorality“) des Unternehmens nicht mehr haltbar (vgl. Paine 2003).

Dieses heutige Verständnis des Unternehmens als sogenannten ,moralischen Akteur' stellt eine wichtige Entwicklung der Unternehmensethik dar, die aber noch von zahlreichen Wissenschaftlern und Praktikern nicht zur Kenntnis genommen worden ist. Es wäre interessant, Richs Meinung dazu zu hören. Er hat sich ja schon in den sechziger und siebziger Jahren, insbesondere im Kontext der Mitbestimmung, mit unternehmensethischen Fragen beschäftigt (vgl. Rich 1973). Obschon mir keine schriftlichen Äußerungen von Rich zum moralischen Status des Unternehmens bekannt sind, dürfen wir annehmen, dass sich Rich dazu eine gründliche Meinung bilden würde (vgl. Rich 1990: 232).

Wie man aus den Medien erfahren und in unzähligen wissenschaftlichen Publikationen lesen kann, umfasst die Unternehmensethik eine unabsehbare Fülle von Fragen und Herausforderungen. Als Beispiele möchte ich nur ein paar wenige herausgreifen, die im Global Compact der Vereinten Nationen genannt sind. ${ }^{3}$ Basierend auf den wichtigsten internationalen Abmachungen, enthält diese Erklärung zehn

3 Siehe http://www.globalcompact.org. 
Unternehmensgrundsätze, zu denen sich Unternehmen freiwillig verpflichten können. Zur Zeit sind es etwa 6000 Organisationen aus allen Kontinenten, die diese Verpflichtung eingegangen sind. Die Grundsätze verlangen von den Unternehmen, die Menschenrechte in ihrem Einflussbereich zu schützen und nicht mitschuldig an deren Verletzung zu werden. Sie sollen überdies zentrale Rechte in der Arbeitswelt schützen, verantwortlich mit der Umwelt umgehen und Korruption bekämpfen. Was diese hohen Grundsätze konkret bedeuten, kann dann - auch auf freiwilliger Basis mit Hilfe der Standards der Global Reporting Initiative genauer bestimmt, gemessen und öffentlich ausgewiesen werden. ${ }^{4}$

Natürlich werfen solche Initiativen viele kritische Fragen auf. Sie machen aber zumindest deutlich, dass Unternehmen offensichtlich Handlungsspielräume haben, die sie verantwortlich ausnutzen können und - ethisch betrachtet - auch sollen. Rich würde sicher diese Auffassung teilen und solche Initiativen kritisch und konstruktiv unterstützen.

\section{Zur Relevanz des zweiten Bandes Marktwirtschaft, Planwirtschaft, Welt- wirtschaft}

Als die englische Übersetzung von Richs Wirtschaftsetbik 2006 veröffentlicht wurde, unternahmen einige meiner Kollegen und ich verschiedene Versuche, Richs Werk in den USA zu verbreiten. Aber leider waren wir nicht so erfolgreich, wie wir es gewünscht hatten. Vielleicht kam die Thematik der Ethik des Kapitalismus für die Amerikaner etwas zu früh. Über viele Jahre hinweg wurde die Ethik des Wirtschaftssystems in Amerika kaum diskutiert, mit Ausnahme von einigen Wirtschaftsethikern wie Richard De George. In Kontinentaleuropa hingegen war diese Thematik seit den fünfziger Jahren fast unausweichlich und ein Dauerthema, standen sich doch auf relativ engem Raum zwei sehr verschiedene Systeme gegenüber. Erst in den letzten Jahren und noch verstärkt durch die letzte Finanzkrise hat in den USA eine breitere Diskussion über den Kapitalismus begonnen. Stellvertretend sei das Center on Capitalism and Society genannt, an dem berühmte Ökonomen wie Edmund Phelps, Jeffrey Sachs, Amartya Sen und Joseph Stiglitz engagiert sind. ${ }^{5}$

Gleichwohl hat die Diskussion meines Erachtens noch kein besonders hohes Niveau erreicht. So werden Fragen von ,Business and Society' gewöhnlich als Fragen der Unternehmen in der weiten Gesellschaft thematisiert, ohne dass über die Bedeutung des Wirtschaftssystems reflektiert würde. Auch viele Beiträge zu CSR (ein übrigens unklarer Begriff) ignorieren die Makro-Ebene des Wirtschaftssystems und springen

$4 \quad$ Siehe http://www.globalreporting.org.

$5 \quad$ Man kann sich fragen, ob noch andere Gründe für die mangelnde Rezeption von Richs Werk maßgeblich waren. Sicher gibt es verschiedene Gründe, die zu prüfen sind. Eine Kritik, insbesondere im deutschsprachigen Raum, nennt eine nur sehr bedingte Anschlussfähigkeit des Richschen Ansatzes an die zeitgenössische ökonomische Tradition. Es trifft zu, dass sich Rich, der seinen zweiten Band in den 80er Jahren verfasste, nicht mit der ,agency theory', ,transaction cost theory' und ,organizational theory' auseinandersetzte. Er beschäftigte sich aber intensiv mit den Werken prominenter Wirtschaftswissenschafter in Nordamerika (J. K. Galbraith, M. Friedman, P. A. Samuelson) und Europa (H. C. Binswanger, K. P. Hensel, H. Kleinewefers, H. Leipold, A. Müller-Armack, O. Sik). Die Kritik der mangelnden „Anschlussfähigkeit“ scheint deshalb höchstens teilweise berechtigt zu sein. 
von der Meso-Ebene des Unternehmens direkt auf die Makro-Ebene des gesamten gesellschaftlichen Systems, das nicht nur Wirtschaft, sondern auch Politik, Kultur und Umwelt umfasst. Im Folgenden möchte ich an einigen Beispielen aufzeigen, wie Richs Ethik des Wirtschaftssystems die Kapitalismusdiskussion klären und bereichern kann.

\subsection{Es gibt verschiedene Arten von Marktwirtschaft.}

Es ist auffallend, dass Rich zur Bezeichnung unseres Wirtschaftssystems den Begriff der Marktwirtschaft verwendet, wogegen man im angelsächsischen Raum praktisch immer nur von ,capitalism` redet. Er trägt damit dem Umstand Rechnung, dass wir im Deutschen mit ,Marktwirtschaft ${ }^{`}$ einen viel positiveren Klang assozieren, während ,Kapitalismus' schriller tönt und an rücksichtslose Kapitalinteressen erinnern mag. Neben dieser semantischen Nuance hebt natürlich der Begriff der Marktwirtschaft die Art der Koordination wirtschaftlicher Transaktionen hervor, während ,Kapitalismus Eigentum und die damit verbundene Entscheidungsbefugnis betont. Um diese Unterschiede $\mathrm{zu}$ wahren, haben wir deshalb in der englischen Übersetzung den Ausdruck der ,market economy' gebraucht.

Für Rich ist die Unterscheidung von Wirtschaftssystem und Wirtschaftsordnung von großer Bedeutung, weil sie die notwendigen Differenzierungen erlaubt, die leider in der Kapitalismusdebatte häufig unbeachtet bleiben. Was heißt es schon, dass nach dem Fall der Berliner Mauer im Jahr 1989 der ,Triumph des Kapitalismus' ausgerufen und so getan wurde, als ob es nur eine Art der Marktwirtschaft gäbe? Nach Rich ist das Wirtschaftssystem eine grundsätzliche Kategorie, bei der man sich für Markt oder Plan entscheiden muss und keinen ,Dritten Weg' wählen kann. Sie ist aber nicht sozusagen frei stehend, sondern muss sich immer in der einen oder anderen Wirtschaftsordnung konkretisieren. So gibt es eine ,kapitalistische' und eine ,soziale' Marktwirtschaft. Die angelsächsische Marktwirtschaft unterscheidet sich von der nordischen und der japanischen, und neue Arten der Marktwirtschaft können entwickelt werden, zum Beispiel - wofür Rich argumentiert - eine ,sozial-ökologische' oder ,sozial verantwortbare' Marktwirtschaft. Es ist beeindruckend, mit welcher Klarsicht Rich schon vor 20 Jahren ein differenziertes Verständnis von Wirtschaftssystem und Wirtschaftsordnung aufgezeigt hat. Zur Debatte steht nicht ,Kapitalismus: ja oder nein?‘, sondern: Welche Art von Marktwirtschaft sollen wir entwickeln, im nationalen wie im internationalen Kontext?

\subsection{Die ökologische Dimension gehört zu jeder Marktwirtschaft.}

Die Frage nach der Art von Marktwirtschaft ist inzwischen auch im angelsächsischen Raum vermehrt diskutiert worden. Als Beispiele seien das Buch von Adair Turner mit dem interessanten Titel Just Capitalism. The Liberal Economy (2001) und das neulich erschienene und recht erfolgreiche Buch von William Baumol, Robert Litan und Carl Schramm Good Capitalism, Bad Capitalism (2007) genannt. Dass nun zentrale Fragen des Wirtschaftssystems direkt und differenzierter behandelt werden, ist sicher an der Zeit und begrüßenswert. Ich kann aber nicht verstehen, dass dabei die ökologische Herausforderung immer noch ignoriert wird. Für Turner existiert diese Herausforderung offensichtlich nicht, und Baumol und seine Mitautoren erwähnen das Problem der Nachhaltigkeit mit keinem Wort; das Wort ,sustainability` findet sich 
in deren Stichwortverzeichnis nicht und die Thematik, wie sie von der Weltkommission für Umwelt und Entwicklung 1987 definiert worden ist, wird weder angezeigt noch behandelt.

Im starken Gegensatz dazu hat Rich schon in seinem ersten Band von 1984 die Umweltproblematik mit dem Kriterium der Mitgeschöpflichkeit grundlegend mit einbezogen. In seinem zweiten Band von 1990 wurde deren Relevanz und Tragweite für das Wirtschafssystem und die Wirtschaftsordnung eingehend diskutiert und systematisch entfaltet. Es sollte dabei erinnert werden, dass Richs Sensitivität für Umweltfragen maßgeblich von Professor Hans-Christoph Binswanger von der Hochschule St. Gallen beeinflusst wurde, mit dem er den gesamten Text des zweiten Bandes fortlaufend besprach. Das frühzeitige Umweltbewusstsein im deutschsprachigen Raum in den achtziger Jahren wird nun auch zunehmend zu einem wichtigen Thema in den USA, nachdem es dort unter dem Einfluss der Reagan-Administration lange Zeit verdrängt und verharmlost wurde.

\subsection{Regulierungen sind notwendig und müssen den Kriterien des Menschengerechten folgen.}

Rich macht die wichtige Unterscheidung von Regulierung und Reglementierung. Regulierungen definieren die Grundregeln der Marktwirtschaft und müssen deshalb klar und wesentlich sein und auch durchgesetzt werden. Demgegenüber sind Reglementierungen Vorschriften, die bis in alle Einzelheiten das wirtschaftliche Handeln bestimmen wollen. Diese Unterscheidung kommt der im Englischen verwendeten Unterscheidung von ,principle-based' und ,rule-based' Vorschriften nahe. Während Regulierungen für eine wohlfunktionierende Marktwirtschaft notwendig sind, sind Reglementierungen schädlich.

Es ist nun interessant, die globale Finanzkrise im Lichte dieser Unterscheidung zu betrachten. Offenbar ignorierte Alan Greenspan, Chairman der Federal Reserve Bank, diese Differenzierung und setzte Regulierung mit Reglementierung gleich. Wie er in einem Kongress-Hearing zugestand (vgl. Wessel 2009: 65-66), hatte er die Regulierung der Investitionstätigkeit der Finanzinstitute für unnötig, ja schädlich, gehalten, dies in der fälschlichen Annahme, dass die konsequente Verfolgung des Selbstinteresses die Finanzinstitute vor ihrem eigenen Kollaps bewahren würde. Greenspan war bekanntlich ein überzeugter Anhänger der libertären Philosophie von Ayn Rand (1905-1982), die den ,ethischen Egoismus' gepredigt hatte. Hätte er Richs Kriterien der Humanität und besonders das der Relationalität ernst genommen, hätte er das Selbstinteresse nicht absolut setzen und den Ruf nach Regulierung missachten können (siehe Abbildung 3). Es besteht kein Zweifel, dass auch McCloskey Greenspans mangelhafte ethische Grundlage der bloßen Klugheit scharf kritisieren und anstatt dessen alle sieben ,bürgerlichen Tugenden' als Basis für Regulierungen fordern würde.

\section{Alan Greenspan im Kongress-Hearing vom 23. Oktober 2008 (Quelle: Wessel 2009)}

"I made a mistake in presuming that the self-interest of organizations, specifically banks and others, were such that they were best capable of protecting their own shareholders 
and their equity in the firms."

„So the problem here is something which looked to be a very solid edifice, and indeed a critical pillar to market competition and free markets, did break down. And I think that, as I said, shocked me (...) I still do not fully understand why it happened."

Waxman pressed Greenspan: „In other words, you found that your view of the world, your ideology was not right. It was not working."

„Precisely“, Greenspan replied. „That's precisely the reason I was shocked because I had been going for forty years or more with very considerable evidence that it was working exceptionally well.“

Abbildung 3: Alan Greenspan im Kongress-Hearing vom 23. Oktober 2008

(Quelle: Wessel 2009)

\subsection{Die Weltwirtschaft muss partizipativ strukturiert werden und verlangt entsprechende Regulierung.}

Zum Abschluss seines zweiten Bandes gibt Rich einen ,weltwirtschaftlichen Ausblick', der durch außergewöhnliche Klarsicht und Ausgewogenheit besticht. Zwar verwendet Rich den Begriff der Globalisierung nicht (wohl weil dieser Ausdruck um 1990 noch kaum gebraucht wurde). Was aber mit (wirtschaftlicher) Globalisierung gemeint ist nämlich die Entgrenzung der nationalen Märkte zu einem weltweiten Markt mit seinen globalen Interdependenzen (vgl. Rich 1990: 345), stellt für Rich eine gewaltige Herausforderung von weitreichenden Konsequenzen über viele Jahrzehnte dar. Im Kern geht es darum, ob die Rahmenbedingungen der Weltwirtschaft konfrontativ oder partizipativ gestaltet werden. Auf der Basis einer globalen Marktwirtschaft ist partizipativ strukturierte Kooperation nur möglich, wenn diese entsprechend reguliert wird und nicht einem Laissez-faire überlassen bleibt (vgl. Rich 1990: 367). Rich entwickelt dafür eine Reihe von Maximen, die nach wie vor von großer Bedeutung für die Globalisierungsdebatte sind. Ich möchte nur zwei kurz erwähnen, die für die schleppenden Verhandlungen der Doha-Runde der Welthandelsorganisation und die enttäuschenden Ergebnisse des UN-Klimagipfels von Kopenhagen außerordentlich wichtig sind:

- Verzicht der Industriestaaten auf protektionistische Handelshemmnisse, die den konkurrenzfähigen Produkten aus der Dritten Welt den Zugang zu den Märkten erschweren oder gar verunmöglichen;

- Solidarische Umweltpolitik seitens der vermögenden Nationen, die mittels finanzieller und technologischer Subsidien die Entwicklungsländer überhaupt erst instand setzen, ihre ökologischen Probleme von weltweiter Relevanz wirksam anzugehen.

\section{Kurzer Ausblick}

Mit diesem ,Ausblick' möchte ich meine Ausführungen schließen. Arthur Rich war ein Vorausdenker, der mit seiner Weitsicht und Gründlichkeit eine solide Grundlage für die Wirtschaftsethik schuf und Orientierungen erarbeitete, die auch heute und für die absehbare Zukunft von entscheidender Bedeutung sind. In seiner Bescheidenheit hat 
er keine ,Schule' gegründet. Aber sein persönliches Vorbild und sein grundlegendes Werk haben einen konstruktiven Weg aufgezeigt, wie die Wirtschaftsethik weiter entwickelt werden sollte. Manche Vorschläge sind inzwischen von der internationalen Diskussion aufgenommen worden; aber noch viele Einsichten und Perspektiven in Richs Werk warten darauf, von der heutigen Generation von Wirtschaftsethikern und Wirtschaftsethikerinnen fruchtbar gemacht und von einer großen Zahl von Engagierten in Kirche, Wirtschaft und Gesellschaft in die Wirklichkeit umgesetzt zu werden. Arthur Rich hatte die Kompetenz und den Mut, für eine sozial verantwortbare Marktwirtschaft zu kämpfen. Wir ehren ihn am besten, wenn wir auch mutig und kompetent in seinem Sinne handeln.

\section{Literaturverzeichnis}

Baumol, J. W./ Litan, R. E./ Schramm, C. J. (2007): Bad Capitalism, Good Capitalism, and the Economics of Growth and Prosperity, New Haven: Yale University Press.

Brenkert, G. G./ Beauchamp, T. L. (2010): The Oxford Handbook of Business Ethics, Oxford: Oxford University Press.

Cortina, A./ Garcia-Marza, D./ Conill, J. (Eds.) (2008): Public Reason and Applied Ethics: The Ways of Practical Reason in a Pluralist Society, Hampshire, UK: Ashgate.

dtv-Lexikon in 24 Bänden (2006): Wirtschaftsethik, Band 24, 132.

Enderle, G. (2003): Business Ethics, in: Bunnin, N. / Tsui-James, E. P. (Eds.): The Blackwell Companion to Philosophy, Second Edition, Oxford: Blackwell Publishers, 531-551.

Enderle, G./ Homann, K./ Honegger, M./ Kerber, W./ Steinmann, H. (Hrsg.) (1993): Lexikon der Wirtschaftsethik, Freiburg: Herder.

Korff, W./ Feldhaus, S. (Gesamtleitung) (1999): Handbuch der Wirtschaftsethik, Band 1-4, Gütersloh: Mohn.

MacIntyre, A. (1981): After Virtue. A Study in Moral Theory, Notre Dame: University of Notre Dame Press.

McCloskey, D. N. (2006): The Bourgeois Virtues. Ethics for an Age of Commerce, Chicago: University of Chicago Press.

Melé, D. (2008): Business Ethics: Europe Versus America, in: Flynn, G. (Ed.): Leadership and Business Ethics, New York: Springer, 13-27.

Paine, L. S. (2003): Value Shift: Why Companies Must Merge Social and Financial Imperatives to Achieve Superior Performance, New York: McGraw-Hill.

Putnam, H. (2002): The Collapse of the Fact-Value Dichotomy and Other Essays, Cambridge, MA: Harvard University Press.

Rich, A. (1973): Mitbestimmung in der Industrie. Probleme - Modelle - Kritische Beurteilung. Eine sozialethische Orientierung, Zürich: Flamberg.

Rich, A. (1984): Wirtschaftsethik. Grundlagen in theologischer Perspektive, Band I, Gütersloh: Mohn.

Rich, A. (1990): Wirtschaftsethik. Marktwirtschaft, Planwirtschaft, Weltwirtschaft aus sozialethischer Sicht, Band II, Gütersloh: Mohn.

Sen, A. (1999): Development as Freedom, New York: Knopf.

Turner, A. (2001): Just Capital. The Liberal Economy, London: Macmillan.

Wessel, D. (2009): In Fed We Trust. Ben Bernanke's War on the Great Panic, New York: Crown Business. 


\section{Ausgewählte Werke von Arthur Rich}

Rich, A. (1973): Mitbestimmung in der Industrie. Probleme - Modelle - Kritische Beurteilung. Eine sozialethische Orientierung, Zürich: Flamberg.

Rich, A. (1984): Wirtschaftsethik. Grundlagen in theologischer Perspektive (Band I), Gütersloh: Mohn.

Rich, A. (1990): Wirtschaftsethik: Marktwirtschaft, Planwirtschaft, Weltwirtschaft aus sozialethischer Sicht, Band II, Gütersloh: Mohn.

Rich, A. (1993): Etica economica (italienische Übersetzung), Brescia: Queriniana.

Rich, A. (1994): Ethique économique (französische Übersetzung), Genf: Editions Labor et Fides.

Rich, A. (1994): Etika hospodářství (tschechische Übersetzung), Prag: ISE.

Rich, A. (1996): Chozjajstvennaja etika (russische Übersetzung), Moskau: Posev.

Rich, A. (2006): Business and Economic Ethics: The Ethics of Economic Systems (englische Übersetzung), Leuven: Peeters.

\section{Veröffentlichungen zu Arthur Richs Person und Werk}

Edel, S. (1998): Wirtschaftsethik im Dialog. Der Beitrag Arthur Richs zur Verständigung zwischen Theologie und Ökonomie, Stuttgart: Calwer.

Edel, S. (1999): Das Zuordnungsverhältnis von Ethik und Ökonomik als Grundproblem der Wirtschaftsethik im neueren theologischen Kontext. Arthur Rich: Integrative Wirtschaftsethik mit Vorbehalt. Anliegen und Ansatz, in: Korff, W./ Feldhaus, S. (Hrsg.): Handbuch der Wirtschaftsethik, Band 1, Gütersloh: Mohn, 871-876.

Enderle, G. (2006): Introduction to Arthur Rich's Business and Economic Ethics, in: Rich, A.: Business and Economic Ethics: The Ethics of Economic Systems (englische Übersetzung), Leuven: Peeters, XV-XXIII.

Peter, H.-B. (2005): Arthur Rich (1910-1992), in: Lienemann, W./ Mathwig, F. (Hrsg.): Schweizer Ethiker im 20. Jahrhundert. Der Beitrag theologischer Denker, Zürich: Theologischer Verlag Zürich, 149-177.

Wolf, W. (2009): Für eine sozial verantwortbare Marktwirtschaft. Der Wirtschaftsethiker Arthur

Rich, Zürich: Theologischer Verlag Zürich. 